\title{
Magnitude of fear as a function of expected time to an aversive event*
}

\author{
J. D. LaBARBERA $\dagger$ and RUSSELL M. CHURCH \\ Brown University, Providence, Rhode Island 02912
}

\begin{abstract}
Three rats were trained to press a lever on a random-interval $1 \mathrm{~min}$ schedule of food reinforcement. In successive phases of the experiment, electric shocks were superimposed at 1-min fixed intervals, 2-min fixed intervals, or at 1-min random intervals. In the fixed-interval conditions, there was a steep gradient of reduction in response rate as the time for the next shock approached; in the random-interval condition, the response rate following a shock was relatively constant. The present method appears to be adequate to monitor the instantaneous level of fear. The results suggest that animals were timing in units proportional to the fixed interval and that aversive events which occur randomly in time are perceived as phenomenologically random by the rat.
\end{abstract}

Is a schedule of aversive events which is objectively random in time perceived as phenomenologically random by a rat? An answer to this question assumes that the magnitude of fear can be monitored continuously through time. Then, if the aversive events are perceived as occurring randomly in time, the magnitude of fear should be independent of the time since the last occurrence of an aversive event.

Various methods have been used to describe the magnitude of the fear response. The validity of direct measures of autonomic functions, e.g., heart rate, is doubtful, since such measures are markedly affected by motor effort (Black, 1971). Behavioral techniques have also been used to obtain indices of the level of fear. These include the method of acquired drive, secondary punishment, and the conditioned emotional response (Church, 1971). As they have typically been used, these behavioral methods provide measures of the average level of fear during an extended period of time (e.g., during a 3-min signal). But they do not serve to monitor the level of fear continuously.

The basic assumption of the conditioned emotional response method of assessing the magnitude of fear is that a change in the mean response rate reflects a change in the magnitude of fear. In support of the assumption, changes in parameters which should increase fear (e.g., severity of the aversive event) do increase the magnitude of response suppression (Annau \& Kamin, 1961). Presumably, the instantaneous response rate could serve as a continuous indicator of the level of fear.

The first purpose of the present experiment was to validate the method for monitoring instantaneous levels of fear. The method consisted of presenting brief electric shocks to a rat that was pressing a lever for food reinforcement. In the first phase of the present

*This investigation was supported by Research Grant MH-19704 from the National Institute of Mental Health. Requests for reprints should be sent to R.M. Church, Department of Psychology, Brown University, Providence, Rhode Island 02912 .

+Now at Vanderbilt University, Nashville, Tennessee. experiment, the aversive event was repeatedly presented at fixed intervals of $1 \mathrm{~min}$. This should lead to the temporal conditioning of fear, which should be reflected in a gradual decrease in response rate as the time of the next shock approached. This situation is simpler than the typical conditioned emotional response experiment in which additional external stimuli are correlated with the occurrence of the aversive event. To demonstrate that the rats were anticipating the next shock rather than reacting to the previous one, temporal gradients with a 2 -min intershock interval were also measured. The response rate of a purely reactive animal would be the same function of time from the last shock in the 1-min and 2-min conditions, but the response of an anticipatory $S$ would be functionally related to the expected time to the next shock.

The second purpose of the experiment was to identify the units of timing employed by the rats during temporal conditioning. If the animals were using absolute units (e.g., seconds), the response rate would be a function of the number of seconds remaining to shock; if the animals were using relative units (e.g., proportional parts of the interval), the response rate would be a function of the proportion of the fixed interval. In Sidman avoidance conditioning there is some evidence that animals are timing in proportional parts of the interval between response and shock (Gibbon, 1971, 1972).

The final purpose of the experiment was to determine the level of fear as a function of time since the previous aversive event when presented on a random schedule. If the response rate monitors the level of fear and if there is an equivalence between objective and subjective randomness, the response rate would be unrelated to the time of occurrence of a random shock.

\section{METHOD}

\section{Subjects}

The Ss were three male albino Norway rats (Charles River $\mathrm{CD}$ ). They were experimentally naive and 64 days old at the 

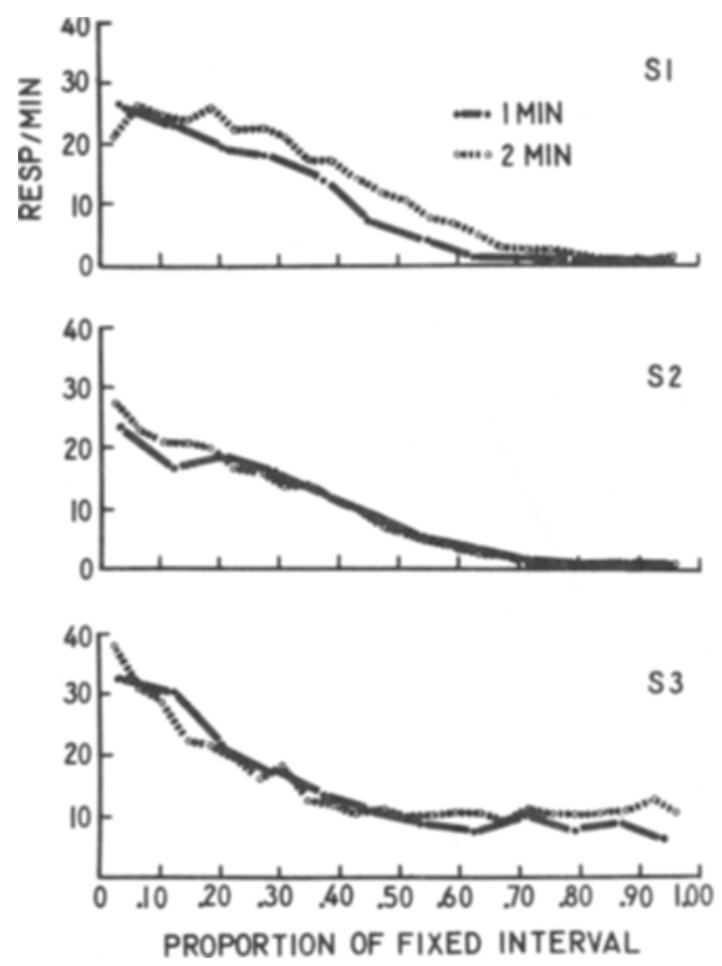

Fig. 1. Mean response rate of each of the three Ss as a function of proportion of fixed interval between successive shocks. The interval between shocks was fixed at $1 \mathrm{~min}$ or $2 \mathrm{~min}$ (Phase 2).

start of training. Throughout the experiment, each rat received a daily $14 \mathrm{~g}$ tation of ground Purina chow mixed with about $25 \mathrm{cc}$ water. (The data from a fourth $S$ are not reported. That rat was relatively insensitive to variations in the experimental procedures in later sessions of the experiment.)

\section{Apparatus \\ Four lever boxes $(23.2 \times 20.3 \times 19.1 \mathrm{~cm}$ high) were used in the experiment. The roof and the two side panels were made of transparent acrylic; the front and back walls were aluminum. The chamber floor was composed of 16 parallel stainless steel bars. Electric shocks were delivered to the grid through an autotransformer, isolation transformer, and $150 \mathrm{~K}-\mathrm{ohm}$ series resistance. A retractable stainless steel lever $(1.3 \times 5.1 \mathrm{~cm})$ projected through the front panel, $5.1 \mathrm{~cm}$ above the floor. A pellet dispenser (Gerbrands Model D-1) delivered 45-mg Noyes Precision food pellets through an opening in the front panel to a food tray. A 6-W houselight was attached to the exterior of the roof of each lever box. Each lever box was housed in a large insulation board chamber designed to attenuate sounds and block visual stimuli. Each chamber was also equipped with a blower and an acrylic window. Implementation of the procedure and collection of data were controlled by a time-shared PDP-12 computer.}

\section{Procedure}

All sessions were $50 \mathrm{~min}$ in length. At the start of each session, the lever was inserted and the houselight was illuminated. At the end of each session, the lever was withdrawn and the houselight turned off.

Pretraining. During the first session, food was delivered on the average of once per minute on a random schedule. (The lever was not inserted on this session of magazine training.) On the next two sessions all lever responses were reinforced. During the next 12 sessions, the lever responses were reinforced on a 1-min random-interval schedule such that a reinforcement would be primed on the average of once a minute, and it would be delivered immediately following the next lever response. The mean response rates of the three rats during the last three sessions of pretraining were 14,16 , and 26 responses $/ \mathrm{min}$.

Training. The 1-min random-interval schedule of food reinforcement remained in effect during the sessions of training. An attempt was made to suppress and maintain the response rate of each $S$ to approximately one-half its rate during the last three sessions of pretraining. Thus, the target response rates were 7,8 , and 13 responses/min for the three Ss. Shock intensity was adjusted between sessions and shock duration was adjusted within sessions to accomplish this goal. On the first session of training the severity of shock for all Ss was $75 \mathrm{~V}$ for $0.2 \mathrm{sec}$. Shock duration was increased by $0.1 \mathrm{sec}$ when the response rate of the previous minute was above the criterion; shock duration was decreased by $0.1 \mathrm{sec}$ (to a minimum of $0.1 \mathrm{sec}$ ) when the response rate of the previous minute was equal to or below the criterion. Shock intensity was adjusted at the beginning of each session according to the following rules: (1) If the final duration of the previous session was at the minimum $(0.1 \mathrm{sec})$, shock intensity was decreased by $15 \mathrm{~V}$ for the session and initial duration was set at $1.0 \mathrm{sec}$; (2) if the final duration for the previous session was greater than 0.1 and did not exceed $1.0 \mathrm{sec}$, the shock intensity and duration of the final shock was used for the first shock of the next session; (3) if the final duration for the previous session exceeded $1.0 \mathrm{sec}$, shock intensity was increased $15 \mathrm{~V}$ for the next session and duration was set at $0.1 \mathrm{sec}$. The treatment conditions were as follows: Phase 1 (27 sessions), 1-min fixed interval: The shock was delivered at the end of the first minute and every $60 \mathrm{sec}$ thereafter throughout the 50-min session. Phase 2 ( 10 sessions), 2 -min fixed interval: The shock was delivered at the end of the second minute and every $120 \mathrm{sec}$ thereafter throughout the 50 -min session. Phase 3 (5 sessions), no-shock control: No shock occurred during the session. Phase 4 (10 sessions), 1-min fixed interval: This was a replication of the conditions described in Phase 1 . Phase 5 ( 5 sessions), no-shock control: This was a replication of the conditions described in Phase 3. Phase 6 (13 sessions), 1-min random interval: The shocks were delivered to each $S$ on a 1-min random-interval schedule. In this condition, the probability of shock in each . 1 -sec interval was $1 / 600$. Thus, on the average, 50 shocks would occur in a session, but the actual number could be substantially larger or smaller. The time from one shock to the next was an exponential waiting time distribution.

\section{RESULTS}

Figure 1 shows the mean response rate as a function of time since last shock for the three rats with shocks at 1-min and 2-min fixed intervals. The data are the means of the final three sessions of Phases 1 and 2. The time axis is scaled in terms of fractions of the fixed interval to reveal the similarity of the form of the two temporal gradients. In all cases, there was a considerable decrease in the response rate as the time for the next shock approached.

Gradients obtained during the last three sessions of the replication of 1-min fixed-interval shock (Phase 4) were similar to those obtained originally (Phase 1). The response rate of $\mathrm{S} 3$, however, decreased to 1.6 responses/min by the end of the fixed interval. During the last three sessions of Phase 3 (without shock), the mean response rates of the three rats were somewhat higher than during pretraining $(28,15$, and 48 responses/min). Similar rates were obtained during the 
last three sessions of Phase $5(30,16$, and 41 responses/min).

Figure 2 shows the mean response rate as a function of time since last shock for the three rats under the random-interval shock condition. The data are the means of the final three sessions of Phase 6. The time axis is scaled in seconds from the last shock, and it reveals that the response rate was relatively constant with respect to the time since last shock. The least-squares fit to these lines had regression constants of $-.001,-.005$, and .002 , none of which were significantly different from zero.

There were large individual differences in the shock intensity required to achieve the desired reduction in response rate. For example, in the first phase with 1-min fixed-interval shock, the final intensities were 240, 220, and $80 \mathrm{~V}$ for the three rats. The intensities required for criterion suppression under similar conditions increased somewhat later in the experiment. In Phase 4 (the replication of 1-min fixed-interval shock), the final intensities were 330,240 , and $90 \mathrm{~V}$. With random shock (Phase 6) substantially lower intensities were sufficient to achieve criterion suppression $(110,85$, and $60 \mathrm{~V})$.

\section{DISCUSSION}

The method employed in the present experiment serves to monitor the ongoing state of fear of a rat. When the next aversive event continually occurred at a fixed time from some stimulus (the last aversive event), the response rate varied with time from the stimulus. When the next aversive event was independent of any stimulus (the random condition), the response rate was independent of the stimulus.

On the basis of the data from the 1-min fixed-interval condition alone, one could entertain the hypothesis that an observed gradient was actually a burst of responses in reaction to a shock which gradually weakened as a function of time from the shock (Church \& Getty, 1972). The data from the 2-min fixed-interval condition discredit this hypothesis, since the response rate was more closely related to the proportion of the interval than to the time from last shock. Furthermore, no substantial response bursts were observed in the random-interval condition.

Presumably, the animals under conditions of shock occurring at regular intervals of $1 \mathrm{~min}$ or $2 \mathrm{~min}$ were timing the interval. A comparison between the gradient of reduction in response rate when the shocks occurred regularly at $1-\mathrm{min}$ and at 2 -min intervals provides some indication of the nature of this timing. The gradients of reduction in response rate for the 1-min and 2-min fixed intervals were indistinguishable when scaled in terms of the proportion of the interval. This suggests that the animals were not primarily responsive to the number of seconds since the last shock, nor to the number of seconds remaining until the next shock. Apparently, the animals were estimating time, and the units of the

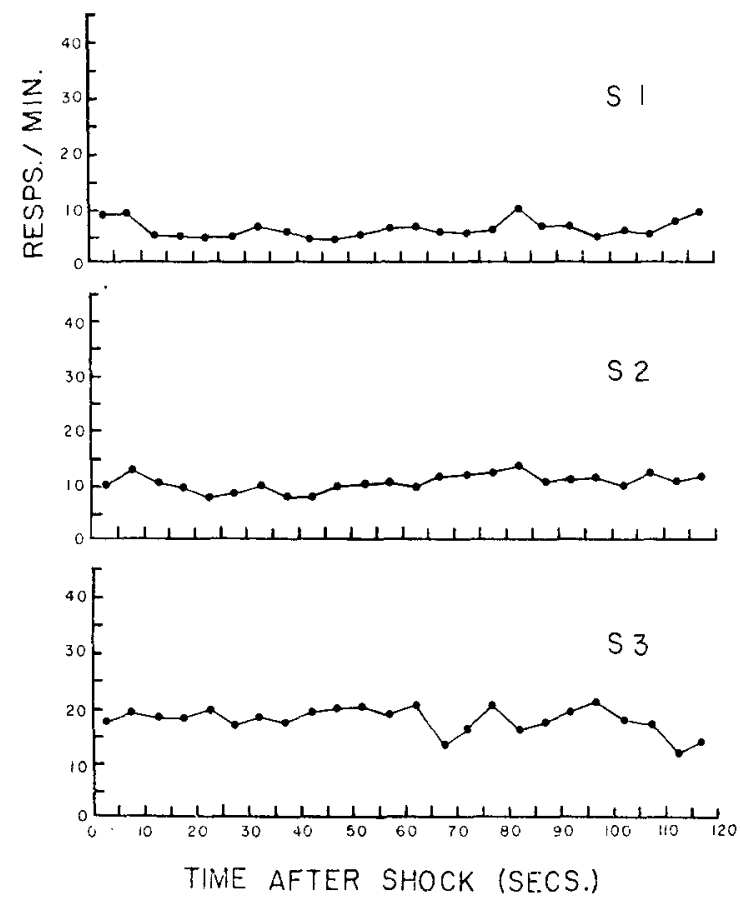

Fig. 2. Mean response rate of each of the three Ss as a function of time since last shock (Phase 6). The intervals between shocks were random intervals with a mean of $1 \mathrm{~min}$.

estimate were proportional parts of the interval between successive shocks.

Previous research has shown that unpredictable shocks are more aversive than predictable shocks (e.g., Seligman, Maier, \& Solomon, 1971). The results of the present experiment are consistent with this finding, since a less intense shock was required to achieve a criterion level of suppression when the shocks were presented at random intervals than at fixed intervals of $1 \mathrm{~min}$.

In monitoring the ongoing state of fear, the procedure served to explore the Ss' conception of randomness. If psychological randomness were divergent from objective randomness, certain consistent changes in fear would have occurred after the delivery of a random shock. This experiment found that no large differences in the magnitude of fear occurred when random shocks were delivered. This finding has important implications for the random control procedure used in research on classical conditioning (Rescorla, 1967). The random control effectively eliminates any objective contingency between CS and US, but it has not been certain that what is random for the $\mathrm{E}$ is random for the $\mathrm{S}$. The observation that response rate was unrelated to the time since last shock would support the notion that an objectively random schedule of shock is phenomenologically random to the rat.

\section{REFERENCES}

Annau, Z., \& Kamin, L. J. The conditioned emotional response as a function of the intensity of the US. Journal of 
Comparative \& Physiological Psychology, 1961, 54, 428-432. Black, A. H. Automonic aversive conditioning in infrahuman subjects. In F. R. Brush (Ed.), Aversive conditioning and learning. New York: Academic Press, 1971. Pp. 3-104.

Church, R. M. Aversive behavior. In J. W. Kling and L. A. Riggs (Eds.), Woodworth and Schlosberg's experimental psychology. 3rd ed. New York: Holt, 1971. Pp. 703-741.

Church, R. M., \& Getty, D. J. Some consequences of the reaction to an aversive event. Psychological Bulletin, 1972, 78, 21-27.

Gibbon, J. Scalar timing and semi-Markov chains in free-operant avoidance. Journal of Mathematical Psychology, 1971, 8, 109-138.
Gibbon, J. Timing and discrimination of shock density in avoidance. Psychological Review, 1972, 79, 68-92.

Rescorla, R. Pavlovian conditioning and its proper control procedures. Psy chological Review, 1967, 74, 71-80.

Seligman, M. E., Maier, S. F., \& Solomon, R. L. Unpredictable and uncontrollable aversive events. In F. R. Brush (Ed.), Aversive conditioning and learning. New York: Academic Press, 1971. Pp. 347-400.

(Received for publication February 12, 1974; revision received April 29, 1974.) 